\title{
THE COMPLEXITY OF CLASS POLYNOMIAL COMPUTATION VIA FLOATING POINT APPROXIMATIONS
}

\author{
ANDREAS ENGE
}

\begin{abstract}
We analyse the complexity of computing class polynomials, that are an important ingredient for CM constructions of elliptic curves, via complex floating point approximations of their roots. The heart of the algorithm is the evaluation of modular functions in several arguments. The fastest one of the presented approaches uses a technique devised by Dupont to evaluate modular functions by Newton iterations on an expression involving the arithmetic-geometric mean. Under the heuristic assumption, justified by experiments, that the correctness of the result is not perturbed by rounding errors, the algorithm runs in time

$$
O\left(\sqrt{|D|} \log ^{3}|D| M\left(\sqrt{|D|} \log ^{2}|D|\right)\right) \subseteq O\left(|D| \log ^{6+\varepsilon}|D|\right) \subseteq O\left(h^{2+\varepsilon}\right)
$$

for any $\varepsilon>0$, where $D$ is the CM discriminant, $h$ is the degree of the class polynomial and $M(n)$ is the time needed to multiply two $n$-bit numbers. Up to logarithmic factors, this running time matches the size of the constructed polynomials. The estimate also relies on a new result concerning the complexity of enumerating the class group of an imaginary quadratic order and on a rigorously proven upper bound for the height of class polynomials.
\end{abstract}

\section{Motivation And Results}

The theory of complex multiplication yields an efficient approach to the construction of elliptic curves over a finite field having a given endomorphism ring of discriminant $D$, as long as $|D|$ is not too large. Exploiting the link between the endomorphism ring of an elliptic curve and its number of points, it is possible to efficiently obtain curves with specific properties. Applications include primality proving [3], the construction of classical, discrete logarithm based elliptic curve cryptosystems and of identity based cryptosystems [36, 19, 5, 7].

The classical approach to effective complex multiplication is to compute minimal polynomials of special, algebraic values of modular functions on the upper complex half-plane (more details are provided in Section 2). The complexity of this algorithm remains a shady issue; one of the reasons a serious analysis has not been undertaken so far is the purported numerical difficulties with the computations with complex floating point numbers, an issue that actually does not arise in practice (cf. the short discussion at the end of Section 77).

In 13 the authors present an algebraic generalisation of the complex multiplication algorithms to $p$-adic fields, which yields the same minimal polynomials. Besides ruling out any possible numerical instabilities, they obtain a complexity of

Received by the editor April 24, 2007 and, in revised form, May 6, 2008

2000 Mathematics Subject Classification. Primary 11Y16, Secondary 11G15.

(c) 2008 by Andreas Enge 
$O\left(|D|^{1+\varepsilon}\right)$, which is better than a straightforward implementation of the complex approach, and asymptotically optimal (up to logarithmic factors) due to the size of the constructed objects.

The present article thus has two goals. First, it provides an accurate account of the complexity of different algorithms for class polynomial computation via floating point approximations. Second, it shows that asymptotically optimal algorithms exist also in the complex setting, with a complexity that is linear (up to logarithmic factors) in the output size. These new algorithms are presented in Sections 6.3 and 6.4, and the faster one achieves the following:

Theorem 1.1. Let $f$ be a fixed modular function that is a class invariant for a family of discriminants $D$ of class numbers $h=h(D)$. Then the algorithm of Section 6.4, which computes a floating point approximation to the class polynomial for $f$, runs in time

$$
O\left(h\left(\log ^{2} h+\log n\right) M(n)\right)
$$

when executed with complex floating point numbers of $n=n(D)$ bits precision, where $M(n)$ is the time needed to multiply two such numbers as detailed in Section 3.1.

The floating point precision $n$ required to carry out the computations is clearly bounded from below by the height of the class polynomial. The following theorem provides a rigorously proven upper bound on the heights, that is close to experimental findings. A bound of the same shape is given in [1, §3.3] as a heuristic, and the gist of the proof already appears in [34, §5.10] and [41, Section 2].

Theorem 1.2. The logarithmic height of the class polynomial for $j$ for the discriminant $D$ of class number $h$ is bounded above by

$$
\begin{aligned}
& c_{5} h+c_{1} N\left(\log ^{2} N+4 \gamma \log N+c_{6}+\frac{\log N+\gamma+1}{N}\right) \\
\leq & c_{1} N \log ^{2} N+c_{2} N \log N+c_{3} N+c_{1} \log N+c_{4}
\end{aligned}
$$

where $N=\sqrt{\frac{|D|}{3}}, \gamma=0.577 \ldots$ is Euler's constant, $c_{1}=\sqrt{3} \pi=5.441 \ldots, c_{2}=$ $18.587 \ldots, c_{3}=17.442 \ldots, c_{4}=11.594 \ldots, c_{5}=3.011 \ldots$ and $c_{6}=2.566 \ldots$

The asymptotic upper bound of

$$
O\left(\sqrt{|D|} \log ^{2}|D|\right)
$$

holds for any other class invariant as well.

In practice, one observes that rounding errors do not disturb the result. It suffices to take $n$ as an approximation of the height plus a few guard digits to be able to round the floating point approximation of the class polynomial to the correct polynomial with integral coefficients. A rigorous error analysis, however, appears to be out of reach. So Theorems 1.1 and 1.2 can be brought together only in the form of a heuristic, assuming that the computations with floating point numbers of $n$ bits yield an approximation of the class polynomial that is correct on essentially $n$ bits.

Corollary 1.3 (heuristic). Taking $n \in O\left(\sqrt{|D|} \log ^{2}|D|\right)$ in Theorem 1.1 and using the bound on the class number $h \in O(\sqrt{|D|} \log |D|)$ proved at the end of Section 4 , the algorithm of Section 6.4 computes the class polynomial for $f$ in time

$$
O\left(\sqrt{|D|} \log ^{3}|D| M\left(\sqrt{|D|} \log ^{2}|D|\right)\right) \subseteq O\left(|D| \log ^{6+\varepsilon}|D|\right) \subseteq O\left(h^{2+\varepsilon}\right)
$$

for any $\varepsilon>0$. 
Notice that up to logarithmic factors, this complexity corresponds to the output size of the algorithm, namely the size of the class polynomials. Notice also that the correctness of the output can be verified by a probabilistic, Monte Carlo type algorithm; namely one may check that the reductions of the class polynomial modulo sufficiently many suitable primes $p$ yield elliptic curves over $\mathbb{F}_{p}$ with complex multiplication by $\mathcal{O}_{D}$. Indeed, the main application of class polynomials is to compute elliptic curves over finite fields with given complex multiplication, and in this situation it can be verified independently that the curves are correct.

As an ingredient for the proof of Theorem 1.1 we obtain in Section 5 the following result for computing class groups of imaginary-quadratic orders:

Theorem 1.4. The class group of the imaginary-quadratic order of discriminant $D$ and class number $h$ can be enumerated:

- unconditionally by a probabilistic algorithm in time

$$
O(\sqrt{|D|} \log |D| \log \log |D| M(\log |D|)) ;
$$

- under GRH by a deterministic algorithm in time

$$
O(h \log \log |D| M(\log |D|)) .
$$

Again the algorithm is essentially optimal in its output size.

\section{Complex multiplication and Class polynomials}

2.1. The basic approach. For proofs of the following facts on complex multiplication of elliptic curves see, for instance, [14.

Let us first consider the situation over the complex numbers. Let $D<0$ be an imaginary quadratic discriminant, and $\mathcal{O}_{D}=\left[1, \frac{D+\sqrt{D}}{2}\right]_{\mathbb{Z}}$ the (not necessarily maximal) order of discriminant $D$ in $K=\mathbb{Q}(\sqrt{D})$. The ideal class number of $\mathcal{O}_{D}$ is denoted by $h=h_{D}$. By Siegel's theorem [42], $\frac{\log h}{\log |D|} \rightarrow \frac{1}{2}(|D| \rightarrow \infty)$, so that $|D| \in O\left(h^{2+\varepsilon}\right)$ and $h \in O\left(|D|^{1 / 2+\varepsilon}\right)$ for any $\varepsilon>0$. There are $h$ isomorphism classes of elliptic curves over $\mathbb{C}$ having complex multiplication by $\mathcal{O}_{D}$, that is, curves with $\mathcal{O}_{D}$ as their endomorphism ring. Namely, let $j: \mathbb{H}=\{z \in \mathbb{C}: \Im(z)>0\} \rightarrow \mathbb{C}$ denote the absolute modular invariant, and let $\tau_{i}=\frac{-B_{i}+\sqrt{D}}{2 A_{i}}$ run through the roots in $\mathbb{H}$ of the reduced quadratic forms $\left[A_{i}, B_{i}, C_{i}\right]=A_{i} X^{2}+B_{i} X+C_{i}$ of discriminant $D=B_{i}^{2}-4 A_{i} C_{i}$, representing the ideal classes of $\mathcal{O}_{D}$; then the $j$ invariants of the elliptic curves are given by the $j\left(\tau_{i}\right)$. Moreover, these $j\left(\tau_{i}\right)$ are algebraic integers; in fact, they generate the so-called ring class field $K_{D}$ for $\mathcal{O}_{D}$, the Galois extension of $K$ whose Galois group is isomorphic to the class group of $\mathcal{O}_{D}$ (the isomorphism being given by the Artin map). The minimal polynomial of the $j\left(\tau_{i}\right)$ over $K, H_{D}(X)=\prod_{i=1}^{h}\left(X-j\left(\tau_{i}\right)\right)$, has in fact coefficients in $\mathbb{Z}$ and is called a class polynomial. In the special case that $D$ is a fundamental discriminant, the ring class field $K_{D}$ is also called the Hilbert class field of $K$.

Now let $\mathbb{F}_{q}=\mathbb{F}_{p^{m}}$ be a finite field of characteristic $p$. Suppose that $p$ splits in $K=\mathbb{Q}(\sqrt{D})$ and that $p \nmid D$; then $p$ is unramified in $K_{D}$. If, furthermore, $q$ may be written as $4 q=U^{2}+D V^{2}$ with $U, V \in \mathbb{Z}$, then the inertia degree of the prime ideals above $p$ in $K_{D}$ divides $m$. The reductions of the complex elliptic curves with complex multiplication by $\mathcal{O}_{D}$ modulo any of these prime ideals thus live in $\mathbb{F}_{q}$. By Deuring's reduction and lifting theorems [16, Einleitung, par. 5], these $h$ curves 
are precisely the elliptic curves over $\mathbb{F}_{q}$ with complex multiplication by $\mathcal{O}_{D}$. They may be obtained as follows: Compute the class polynomial $H_{D} \in \mathbb{Z}[X]$ and reduce it modulo $p$. It splits completely over $\mathbb{F}_{q}$, and each of its roots is the $j$-invariant of an elliptic curve over $\mathbb{F}_{q}$ with the desired endomorphism ring.

2.2. Class invariants. Unfortunately, $H_{D}$ has very large coefficients (see the discussion in Section (4), so that its computation requires a high precision to be accurate. In practice, one may often gain a constant factor for the required number of digits by using instead of $j$ modular functions $f$ that are invariant under $\Gamma^{0}(N)=$ $\left\{\left(\begin{array}{ll}a & b \\ c & d\end{array}\right) \in \mathrm{Sl}_{2}(\mathbb{Z}): N \mid b\right\}$ for some positive integer $N$; that is, $f\left(\frac{a z+b}{c z+d}\right)=f(z)$ for any such matrix $\left(\begin{array}{ll}a & b \\ c & d\end{array}\right)$. Under suitable conditions on the discriminant $D$ and suitable normalisations of the $\tau_{i}$, derived from Shimura's reciprocity law, the singular values $f\left(\tau_{i}\right)$ are still elements of the class field $K_{D}$ [37, 30, 31, 38]; we then call $f$ a class invariant and the minimal polynomial $H_{D}[f](X)=\prod_{i=1}^{h}\left(X-f\left(\tau_{i}\right)\right)$ again a class polynomial. The first such class invariants are given by Weber's functions $\mathfrak{f}, \mathfrak{f}_{1}, \mathfrak{f}_{2}$, $\gamma_{2}$ and $\gamma_{3}$ [4].

Two parameterised families of class invariants are exhibited in 23, where $N$ is the product of two primes and $H_{D}[f] \in \mathbb{Z}[X]$, and [22, where $N$ is prime and $H_{D}[f]$ has coefficients in the maximal order of $\mathbb{Q}(\sqrt{D})$. Again, these class polynomials split completely after reduction in $\mathbb{F}_{q}$. The corresponding elliptic curves may be recovered from some polynomial relationship between $f$ and $j$ : If the modular curve $X_{0}(N)$ has genus 0 , then $j$ is sometimes given by a rational formula in $f$; otherwise it has been suggested in [23] to look for a root of the modular polynomial $\Phi(f, j)$ after reducing modulo $p$ and specialising in the value found for $f$ in $\mathbb{F}_{q}$. (Both cases require that all coefficients be rational to make sense modulo $p$, which holds for all exhibited class invariants.)

\section{Complexity of ARIthmetics}

This section discusses the well-known complexity of the basic multiprecision and polynomial arithmetic underlying the computations.

3.1. Multiprecision floating point arithmetic. Let $M(n)$ be the bit complexity of multiplying two $n$-bit integers. Using Schönhage-Strassen multiplication 39, one has $M(n) \in O(n \log n \log \log n)$. With Fürer's algorithm 26, one has $M(n) \in$ $n \log n 2^{O\left(\log ^{*} n\right)}$, where $\log ^{*} n$ is the number of times the logarithm function has to be applied to $n$ before the result drops below 1 . So with either algorithm, $M(n) \in O\left(n \log ^{1+\varepsilon} n\right)$ for any $\varepsilon>0$.

The four basic arithmetic operations and the square roots of real floating point numbers of precision $n$ have a complexity of $O(M(n))$, the inversion and square roots being realised by Newton iterations as explained in Lemmata 2.2 and 2.3 of 6]. The same article shows that exp, sin and the constant $\pi$ can be computed in $O(\log n M(n))$.

Thus, the four basic operations on complex floating point numbers of precision $n$ can be executed in time $O(M(n))$. Letting $c=\sqrt{\frac{a+\sqrt{a^{2}+b^{2}}}{2}}$ for $a>0$, one obtains $\sqrt{a+b i}=c+\frac{b}{2 c}$ in time $O(M(n))$. Finally, the complex exponential may be reduced to the real exponential and the real sine and cosine functions and is thus computed with complexity $O(\log n M(n))$. 
3.2. Polynomial arithmetic. Concerning operations with polynomials, we assume that a floating point precision of $n$ bits has been fixed and is the same for all input and output polynomials. Multiplying two polynomials of degree $d$ over $\mathbb{C}$ by the FFT takes $O(d \log d)$ multiplications in $\mathbb{C}$, whence it has a complexity of $O(d \log d M(n))$ once the necessary roots of unity have been computed.

The following algorithm ([27, Algorithm 10.3]) obtains a monic polynomial of degree $h$ from its roots by organising the computations in a binary tree.

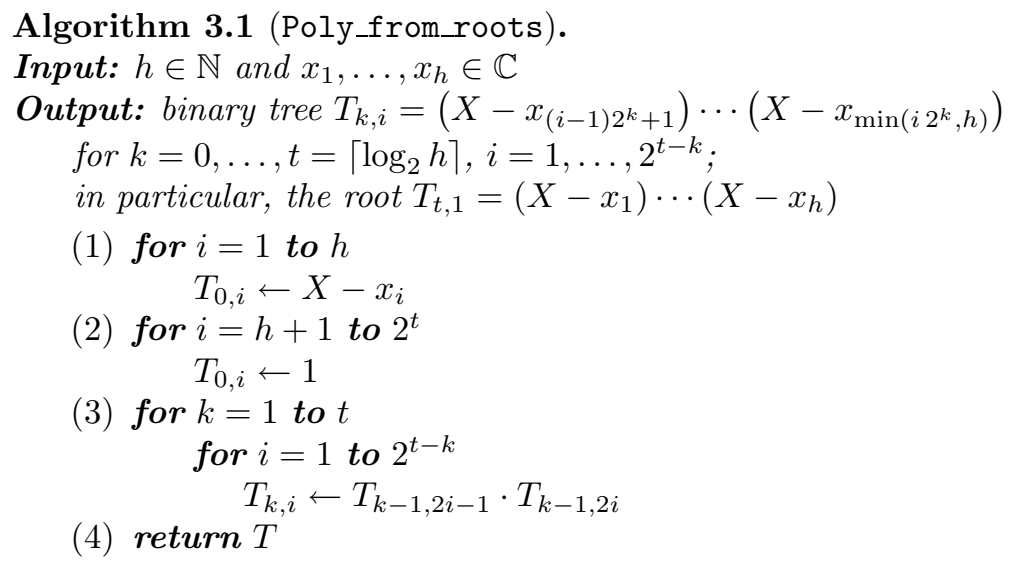

If the needed roots of unity are precomputed, the algorithm has a complexity of $O\left(h \log ^{2} h M(n)\right)$. Notice that the roots of unity of order $2^{\left\lceil\log _{2} h\right\rceil}$ suffice to carry out all the FFTs in Algorithm 3.1. By Section 3.1. computing a primitive root of unity takes $O(\log n M(n))$, all others can be obtained by successive multiplications in $O(h M(n))$. Thus, the total complexity of Algorithm 3.1 becomes

$$
O\left(\left(h \log ^{2} h+\log n\right) M(n)\right) .
$$

One of the asymptotically optimal algorithms for class polynomials relies on a related technique of symbolic computation (see [27, $\S 10.1]$ ). Let $f(X)$ be a polynomial of degree $d$ over $\mathbb{C}$ that is to be evaluated in $h$ different arguments $x_{1}, \ldots, x_{h}$. The key observation is that $f\left(x_{i}\right)$ is nothing but $f(X) \bmod X-x_{i}$. So the first step of the algorithm is the construction of the binary tree $T_{k, i}$ as in Algorithm 3.1 containing products of more and more of the $X-x_{i}$. Then a matching tree $R_{k, i}=f \bmod T_{k, i}$ is computed in the converse order, from its root to its leaves, such that the leaves contain the desired values of $f$.

Algorithm 3.2 (Multi_eval).

Input: $h \in \mathbb{N}, f \in \mathbb{C}[X]$ and $x_{1}, \ldots, x_{h} \in \mathbb{C}$

Output: $f\left(x_{1}\right), \ldots, f\left(x_{h}\right)$

(1) $T \leftarrow$ Poly_from_roots $\left(h, x_{1}, \ldots, x_{h}\right)$

(2) $t \leftarrow\left\lceil\log _{2} h\right\rceil, R_{t, 1} \leftarrow f \bmod T_{t, 1}$

(3) for $k=t-1$ down to 0

$$
\begin{aligned}
& \text { for } i=1 \text { to } 2^{t-k} \\
& \quad R_{k, i} \leftarrow R_{k+1,\left\lfloor\frac{i+1}{2}\right\rfloor} \bmod T_{k, i}
\end{aligned}
$$

(4) return $R_{0,1}, \ldots, R_{0, h}$

A division with remainder of a polynomial of degree $d$ by a polynomial of degree $d^{\prime}$ has the same complexity as multiplying polynomials of degree bounded by $d$ 
([27, §9.1]). The algorithm first computes an inverse modulo some power of $X$ by Newton iterations and obtains the quotient with one multiplication; the remainder then requires another multiplication. So after the first reduction of $f$ modulo the product of all the $X-x_{i}$, the complexity of Algorithm 3.2 on $n$-bit numbers is the same as that of Algorithm 3.1. Including the first reduction, it is given by

$$
O\left(\left(d \log d+h \log ^{2} h+\log n\right) M(n)\right) .
$$

\section{The Height OF ClASS POLYNOMIALS}

The running time of the algorithms working with floating point approximations depends crucially on the precision $n$. To be able to round the approximated class polynomial to a polynomial over the integers $(\mathbb{Z}$ or the maximal order of an imaginary-quadratic number field), $n$ has to be at least the bit size of the largest coefficient, or otherwise said, the logarithmic height of the polynomial. In this section, we prove Theorem 1.2 by developing an explicit upper bound without hidden constants for the height of the class polynomial for $j$. The result is of independent interest; for instance, it allows us to bound the precision also for the $p$-adic algorithms, which yields a proof of correctness for their output.

As shown in [21, the height of class polynomials for a different $f$ changes asymptotically by a constant factor, depending on the degrees in $f$ and $j$ of the modular polynomial connecting the two functions. So this section proves an asymptotic bound of $O\left(\sqrt{|D|} \log ^{2}|D|\right)$ for the height of any class polynomial. To obtain a more explicit bound for invariants other than $j$, the techniques of this section may be used with the necessary adaptations.

Let us first give the intuitive basis for the bound. Since the values of $j$ are usually large, more often than not the largest coefficient of the class polynomial is its constant one. Then approximating $j(\tau)=q^{-1}+744+\sum_{\nu=1}^{\infty} c_{\nu} q^{\nu}$ by the first term, $q^{-1}$, one obtains a heuristic estimate for the height as

$$
\pi \sqrt{|D|} \sum_{[A, B, C]} \frac{1}{A}
$$

where the sum is taken over all reduced primitive quadratic forms of discriminant $D$. This heuristic estimate is very well confirmed by experimental findings; see [21].

Turning this idea into an explicit bound is mainly a matter of computations that are sketched in the following.

The reducedness of the quadratic forms is equivalent to the $\tau$-values lying in the standard fundamental domain $\mathcal{F}$ for the action of $\mathrm{Sl}_{2}(\mathbb{Z})$ on $\mathbb{H}$,

$$
\mathcal{F}=\left\{z \in \mathbb{H}:|z|>1,-\frac{1}{2} \leq \Re(z)<\frac{1}{2}\right\} \cup\left\{z \in \mathbb{H}:|z|=1,-\frac{1}{2} \leq \Re(z) \leq 0\right\} .
$$

Then $|q| \leq\left|e^{2 \pi i \frac{1+\sqrt{-3}}{2}}\right|=e^{-\pi \sqrt{3}}$. The upper bound $c_{\nu} \leq \frac{e^{4 \pi \sqrt{\nu}}}{\sqrt{2} \nu^{3 / 4}}$ of 8 then yields

$$
\left|j(\tau)-q^{-1}\right| \leq 744+\sum_{\nu=1}^{\infty} \frac{e^{4 \pi \sqrt{\nu}}}{\sqrt{2} \nu^{3 / 4}} e^{-\pi \sqrt{3} \nu}=k_{1}=2114.566 \ldots
$$

for $\tau$ in the fundamental domain $\mathcal{F}$, so that

$$
|j(\tau)| \leq\left|q^{-1}\right|+k_{1} \leq k_{2}\left|q^{-1}\right|
$$

with $k_{2}=1+k_{1} e^{-\pi \sqrt{3}}=10.163 \ldots$ 
Let the $A_{i}$ be numbered in increasing order. Then the logarithm of the absolute value of the coefficient in front of $X^{j}$ is bounded above by

$$
\log \left(\left(\begin{array}{l}
h \\
j
\end{array}\right) \prod_{i=1}^{h-j}\left(k_{2}\left|q_{i}^{-1}\right|\right)\right) \leq \log \left(2^{h} k_{2}^{h} \prod_{i=1}^{h}\left|q_{i}^{-1}\right|\right) \leq \log \left(2 k_{2}\right) h+\pi \sqrt{|D|} \sum_{i=1}^{h} \frac{1}{A_{i}}
$$

independently of $j$.

The next step consists of estimating $\sum_{i=1}^{h} \frac{1}{A_{i}}$. It is proved in [41, Lemma 2.2] that $\sum_{i=1}^{h} \frac{1}{A_{i}} \in O\left(\log ^{2}|D|\right)$. We shall derive a bound that makes the involved constants explicit. Consider the number of possible $B$ for a given $A$. This number is certainly bounded above by the number of $B \in]-A, A]$ that satisfy $B=D \bmod 2$ and $\frac{B^{2}-D}{4}=0 \bmod A$. Assuming the worst case that the quadratic equation has a root modulo each prime divisor of $A$ and considering the cases of odd and even $A$ separately, one obtains an upper bound on the number of $B$ of $2 \cdot 2^{\omega(A)} \leq 2 \tau(A)$, where $\omega(A)$ denotes the number of prime factors of $A$ and $\tau(A)$ its number of divisors. Hence,

$$
\sum_{i=1}^{h} \frac{1}{A_{i}} \leq 2 \sum_{A=1}^{\sqrt{\frac{|D|}{3}}} \frac{\tau(A)}{A}
$$

and this sum may be bounded by standard techniques from analytic number theory. We use the estimates

$$
\log N+\gamma \leq \sum_{n=1}^{N} \frac{1}{n} \leq \log N+\gamma+\frac{1}{2 N}
$$

with Euler's constant $\gamma=0.577 \ldots$ and

$$
\sum_{n=1}^{N} \frac{\log n}{n} \geq \frac{\log 2}{2}+\int_{3}^{N+1} \frac{\log t}{t} d t \geq \frac{1}{2} \log ^{2} N-k_{3}
$$

with $k_{3}=\frac{1}{2}\left(\log ^{2} 3-\log 2\right)=0.256 \ldots$ We have

$$
\begin{aligned}
2 \sum_{A=1}^{N} \frac{\tau(A)}{A} & =2 \sum_{A=1}^{N} \sum_{1 \leq m, n: m n=A} \frac{1}{m n}=2 \sum_{1 \leq m, n: m n \leq N} \frac{1}{m n}=2 \sum_{m=1}^{N} \frac{1}{m} \sum_{n=1}^{\lfloor N / m\rfloor} \frac{1}{n} \\
& \leq 2 \sum_{m=1}^{N} \frac{1}{m}\left(\log \frac{N}{m}+\gamma+\frac{1}{2\left\lfloor\frac{N}{m}\right\rfloor}\right) \\
& \leq \log ^{2} N+4 \gamma \log N+2 \gamma^{2}+\frac{\log N+\gamma}{N}+2 k_{3}+\sum_{m=1}^{N} \frac{1}{m\left\lfloor\frac{N}{m}\right\rfloor}
\end{aligned}
$$

by (4.2) and (4.3).

Using (4.2), the last term of this sum can be bounded by

$$
\sum_{m=1}^{N} \frac{1}{m\left\lfloor\frac{N}{m}\right\rfloor} \leq \sum_{m=1}^{\left\lfloor\frac{N}{2}\right\rfloor} \frac{1}{m\left(\frac{N}{m}-1\right)}+\sum_{m=\left\lfloor\frac{N}{2}\right\rfloor+1}^{N} \frac{1}{m} \leq 2 \sum_{m=\left\lceil\frac{N}{2}\right\rceil}^{N} \frac{1}{m} \leq 2 \log 2+\frac{1}{N} .
$$


Combining the inequalities, the height of the class polynomial is bounded from above by

$$
k_{5} h+\pi \sqrt{|D|}\left(\log ^{2} N+4 \gamma \log N+\frac{\log N+\gamma+1}{N}+k_{4}\right)
$$

with $N=\sqrt{\frac{|D|}{3}}, k_{4}=2 k_{3}+2 \log 2+2 \gamma^{2}=2.566 \ldots$ and $k_{5}=\log \left(2 k_{2}\right)=3.011 \ldots$

Similarly to the argumentation above one shows that

$$
h \leq 2 \sum_{A=1}^{N} \tau(A) \leq 2 N(\log N+\gamma)+1,
$$

which also implies $h \in O(\sqrt{|D|} \log |D|)$.

Combining (4.4) and (4.5) yields the bound of Theorem 1.2 .

\section{Class group enumeration}

Computing the class group of $\mathcal{O}_{D}$ by enumerating all reduced primitive quadratic forms of discriminant $D$ is a step of the algorithm that can be neglected in practice. In the new algorithms of Sections 6.3 and 6.4 however, its theoretical complexity risks coming close to that of the crucial parts. One has to determine all coprime $\left[A_{i}, B_{i}, C_{i}\right]$ such that $\left|B_{i}\right| \leq A_{i} \leq C_{i}, B_{i}>0$ if one of the inequalities is not strict, and $D=B_{i}^{2}-4 A_{i} C_{i}$. These conditions imply that $A_{i} \leq \sqrt{\frac{|D|}{3}}$.

5.1. The naïve algorithm. The following trivial algorithm is implemented in the code described in more detail in Section 7 Loop over $A$ and $B$ such that $0 \leq B \leq A \leq \sqrt{\frac{|D|}{3}}$ and $B \equiv D(\bmod 2)$ and compute the corresponding $C$, if it exists. While it can be seen in the figures of Table 1 that this approach takes virtually no time, it carries out $O(|D|)$ arithmetic operations with integers of bit size in $O(\log |D|)$ and thus has a complexity of

$$
O(|D| M(\log |D|))
$$

which is smaller than the bound of Corollary 1.3 only by logarithmic factors.

5.2. Saving one loop. An asymptotically faster (and again up to logarithmic factors optimal) algorithm is inspired by the theoretical considerations of Section 4 .

Looping over $A$ in the interval $1 \leq A \leq \sqrt{\frac{|D|}{3}}$, one solves the congruence $B=$ $D \bmod 2$ and $\frac{B^{2}-D}{4}=0 \bmod A$, and keeps the form if $C=\frac{B^{2}-D}{4 A} \geq A$. As shown in Section 4, the total number of $B$ to consider is in $O(\sqrt{|D|} \log |D|)$.

To find the square root of $D$ modulo $A$, the prime factorisation of $A$ is required, and the most convenient approach is to enumerate the $A$ in factored form. To this purpose, one needs the primes up to $\sqrt{\frac{|D|}{3}}$, which can be obtained in time $O\left(\frac{\sqrt{|D|} \log |D|}{\log \log |D|}\right)$ by [2]. All possible $A$ are then computed with an amortised cost of one multiplication per value in total time $O(\sqrt{|D|} M(\log |D|))$.

For any given $A$ and prime $p \mid A$, a square root of $D$ modulo $p$ is computed in $O(\log p M(\log p))$ by Cipolla's algorithm [11, lifting to a root modulo $p^{e}$ for $p^{e} \| A$ 
requires an additional $O(e M(\log p))$. For one $A$, this takes

$$
O\left(\sum_{p^{e}|| A} \log \left(p^{e}\right) M(\log p)\right) \subseteq O(\log A M(\log A)) \subseteq O(\log |D| M(\log |D|)),
$$

or $O(\sqrt{|D|} \log |D| M(\log |D|))$ for all $A$.

The roots modulo prime powers have to be recombined by the Chinese remainder theorem to form a candidate $B$. Organising the computations in a tree with the leaves indexed by the $p^{e} \| A$, one may use the analogues for numbers of the fast algorithms for polynomials of Section 3.2 . The tree has $\omega(A) \in O(\log A)$ leaves and thus a height of $O(\log \log A)$, and the root contains a number of $O(\log A)$ digits, so that one computation of Chinese remainders takes

$$
O(\log \log A M(\log A)) .
$$

This is to be multiplied by the total number of $B$, which yields an overall complexity of

$$
O(\sqrt{|D|} \log |D| \log \log |D| M(\log |D|)) .
$$

So Chinese remaindering is the dominant step of this algorithm for class group enumeration, and the first bound of Theorem 1.4 is proved.

5.3. Generating the class group by primes. Another possible approach is to start with a set of prime forms generating the class group. According to [4, p. 376], under GRH a system of generators is given by the forms having a prime $A$-value bounded by $6 \log ^{2}|D|$, and these can be computed in time polynomial in $\log |D|$. Let $\mathfrak{p}_{1}, \mathfrak{p}_{2}, \ldots$ denote these generators. One may enumerate the powers of $\mathfrak{p}_{1}$ in the class group by composing and reducing quadratic forms until reaching the order $e_{1}$ of $\mathfrak{p}_{1}$ such that $\mathfrak{p}_{1}^{e_{1}}=1$. Next, one computes the powers of $\mathfrak{p}_{2}$ until $\mathfrak{p}_{2}^{e_{2}}$ lies in the subgroup generated by $\mathfrak{p}_{1}$, constructed in the previous step; then all combinations $\mathfrak{p}_{1}^{a_{1}} \mathfrak{p}_{2}^{a_{2}}$ with $0 \leq a_{i}<e_{i}$ are added to yield the subgroup $\left\langle\mathfrak{p}_{1}, \mathfrak{p}_{2}\right\rangle$. One continues with the powers of $\mathfrak{p}_{3}$ until $\mathfrak{p}_{3}^{e_{3}}$ falls into this subgroup, and so forth. If all computed elements are stored in a hash table or simply in a matrix indexed by $A$ and $B$, looking them up takes negligible time. The running time of the algorithm is dominated by $O(h)$ computations in the class group, each of which takes $O(\log \log |D| M(\log |D|))$ by [40. This proves the second bound of Theorem 1.4.

5.4. From class groups to $N$-systems. When working with a class invariant other than $j$ that is not invariant under $\mathrm{Sl}_{2}(\mathbb{Z})$, but only under $\Gamma^{0}(N)$ for some $N>0$, the representatives of the class group need to be normalised to obtain a coherent set of algebraic conjugates. Such a normalisation is, for instance, given by an $N$-system as defined in 38. It has the property that

$$
\operatorname{gcd}\left(A_{i}, N\right)=1 \text { and } B_{i} \equiv B_{1} \quad(\bmod 2 N) \quad \text { for } 1 \leq i \leq h
$$

and may be obtained by applying suitable unimodular transformations to the original $\left[A_{i}, B_{i}, C_{i}\right]$. The coefficients of these transformations are defined modulo $N$, so that for fixed $N$, trying all possibilities requires a constant number of arithmetic operations per form and increases the size of the $A_{i}, B_{i}$ and $C_{i}$ by a constant factor. Thus, transforming a system of reduced quadratic forms into an $\mathrm{N}$-system requires an additional $O(h M(\log |D|))$, which is covered by the previous enumeration of the forms. (In practice, one would use a more intelligent approach to lower the complexity with respect to $N$; cf. the constructive proof of Proposition 3 in [38.) 


\section{Complexity of Class polynomial COMPUtATion}

We are now able to provide the generic complexity for class polynomial computation via floating point approximations. Different approaches to evaluating the class invariants lead to algorithms with different overall complexities; these are examined below.

Let $n$ be the precision in bits used for the computations, let $h$ be the class number of $\mathcal{O}_{D}$ and denote by $E(h, n)$ the time needed to evaluate the class invariant with precision $n$ in $h$ values. Then the algorithm takes

- $O\left(h^{1+\varepsilon}\right)$ for enumerating the class group according to Section 5 .

- $E(h, n)$ for evaluating the class invariant, and

- $O\left(\left(h \log ^{2} h+\log n\right) M(n)\right)$ for reconstructing the class polynomial from its roots according to Algorithm 3.1.

The class group computation is indeed negligible since $E(h, n)$ is at least of order $h n$, which is the time needed to write down the conjugates. So it remains to examine in more detail the quantity $E(h, n)$.

We propose four different algorithms for evaluating modular functions. The first two are well-known, the third one is a novel application of the techniques of symbolic computation presented in Section [3.2, and it already allows us to obtain the complexity stated in Corollary 1.3. The fourth one gains an additional logarithmic factor for the evaluation phase and yields the slightly more precise statement of Theorem 1.1 without changing the conclusion of Corollary 1.3

The class polynomial $H_{D}[f]=\prod_{i=1}^{h}\left(X-f\left(\tau_{i}\right)\right)$ is obtained by evaluating the function $f$ in the $h$ different arguments $\tau_{i}=\frac{-B_{i}+\sqrt{D}}{2 A_{i}}$ for an $N$-system $\left[A_{i}, B_{i}, C_{i}\right]$ (see Section 3.2), where $N$ depends on $f$ and is assumed to be fixed. Since $f(z)$ is modular for $\Gamma^{0}(N)$, it is invariant under the translation $z \mapsto z+N$ and admits a Fourier transform, that is, a Laurent series expansion in the variable $q^{1 / N}=e^{2 \pi i z / N}$. Thus, the $f\left(\tau_{i}\right)$ may be obtained by first computing the corresponding $q_{i}^{1 / N}$ and then evaluating the $q$-expansion in these arguments. By Section 3.1, the $q_{i}$ can be computed in time $O(h \log n M(n))$, which will be dominated by the actual function evaluations.

6.1. The naïve approach. The straightforward technique for evaluating the modular function $f=\sum_{\nu=\nu_{0}}^{\nu_{1}} c_{\nu}\left(q^{1 / N}\right)^{\nu}$ consists of a Horner scheme for the polynomial part $\sum_{\nu=0}^{\nu_{1}-\nu_{0}} c_{\nu+\nu_{0}}\left(q^{1 / N}\right)^{\nu}$ and a multiplication by $\left(q^{1 / N}\right)^{\nu_{0}}$. (Notice that usually $\nu_{0}<0$.) Its complexity is $O\left(\left(\nu_{1}-\nu_{0}+\log \nu_{0}\right) M(n)\right)$, the $c_{\nu}$ having been precomputed to precision $n$. Actually, $\nu_{0}$ is a constant depending only on $f ; \nu_{1}$, however, depends not only on the $c_{\nu}$, but also on the desired precision $n$ and on $\left|q^{1 / N}\right|$ and thus on the function argument.

Consider first the classical case of $f$ being $j$, which is invariant under $\operatorname{Sl}_{2}(\mathbb{Z})$. We may then assume that the arguments are transformed by a matrix in $\mathrm{Sl}_{2}(\mathbb{Z})$ into the standard fundamental domain $\mathcal{F}$ of (4.1) prior to evaluating $j$, so that $|q| \leq e^{-\pi \sqrt{3}}$ is bounded from above by a constant less than 1 . On the other hand, it is shown in [8] that $0 \leq c_{\nu} \leq \frac{e^{4 \pi \sqrt{\nu}}}{\sqrt{2} \nu^{3 / 4}}$ for $\nu \geq 1$. Thus, it is possible to fix $\nu_{1} \in O(n)$ to obtain a precision of $O(n)$ digits.

The total complexity of evaluating $j$ at $h$ values then becomes

$$
O(h n M(n)),
$$


or

$$
O\left(|D| \log ^{3}|D| M\left(\sqrt{|D|} \log ^{2}|D|\right)\right) \subseteq O\left(|D|^{3 / 2} \log ^{6+\varepsilon}|D|\right)
$$

with $n \in O\left(\sqrt{|D|} \log ^{2}|D|\right)$ and $h \in O(\sqrt{|D|} \log |D|)$ according to Section 4.

Concerning alternative class invariants, unfortunately the fundamental domain for $\Gamma^{0}(N)$ with $N>1$ contains at least one rational number (called a cusp). In such a cusp, $|q|=1$, and the $q$-expansion usually diverges; in a neighbourhood of the cusp, it may converge arbitrarily slowly. In this case, it is possible to use a different expansion in the neighbourhood by transporting the cusp to infinity via a matrix in $\mathrm{Sl}_{2}(\mathbb{Z})$. We will not pursue this discussion, since the approach of Section 6.2 provides a faster and simpler solution for all currently used class invariants.

6.2. Using the sparsity of $\eta$. Virtually all class invariants suggested in the literature are in some way derived from Dedekind's $\eta$-function. This is the case for the Weber functions $\mathfrak{f}(z)=e^{-\pi i / 24} \frac{\eta\left(\frac{z+1}{2}\right)}{\eta(z)}, \mathfrak{f}_{1}(z)=\frac{\eta\left(\frac{z}{2}\right)}{\eta(z)}$ and $\mathfrak{f}_{2}(z)=\sqrt{2} \frac{\eta(2 z)}{\eta(z)}$ already examined in [43], the generalised Weber functions $\mathfrak{w}_{N}(z)=\frac{\eta\left(\frac{z}{N}\right)}{\eta(z)}$ suggested in [22], the double $\eta$ quotients $\mathfrak{w}_{p_{1}, p_{2}}(z)=\frac{\eta\left(\frac{z}{p_{1}}\right) \eta\left(\frac{z}{p_{2}}\right)}{\eta(z) \eta\left(\frac{z}{p_{1} p_{2}}\right)}$ proposed in [23], and even for $j$. In fact, $j$ is most conveniently computed as $j=\left(\frac{\mathfrak{f}_{1}^{24}+16}{\mathfrak{f}_{1}^{8}}\right)^{3}$.

The definition of $\eta$ in [15] is closely related to the partition generating function:

$$
\eta=q^{1 / 24} \prod_{\nu \geq 1}\left(1-q^{\nu}\right)
$$

evaluating the product to precision $n$ requires $O(n)$ arithmetic operations. An expression better suited for computation is given by Euler's pentagonal number theorem [25]:

$$
\eta=q^{1 / 24}\left(1+\sum_{\nu=1}^{\infty}(-1)^{\nu}\left(q^{\nu(3 \nu-1) / 2}+q^{\nu(3 \nu+1) / 2}\right)\right)
$$

Since the occurring exponents are values of quadratic polynomials, the series is very sparse: To reach an exponent of order $O(n)$, only $O(\sqrt{n})$ terms need to be computed, and this process can be implemented with $O(\sqrt{n})$ multiplications. Recall that any polynomial of fixed degree can be evaluated in an arithmetic progression with a constant number of arithmetic operations per additional value, once the first few values are known; the employed algorithm relies on iterated differences. In the special case of $\eta$, the following recursion yields two additional terms of the series at the expense of four multiplications by recursively computing $q^{\nu}, q^{2 \nu-1}, q^{\nu(3 \nu-1) / 2}$ and $q^{\nu(3 \nu+1) / 2}$ as follows:

$$
\begin{aligned}
q^{\nu} & =q^{\nu-1} \cdot q, \\
q^{2 \nu-1} & =q^{2(\nu-1)-1} \cdot q^{2}, \\
q^{\nu(3 \nu-1) / 2} & =q^{(\nu-1)(3(\nu-1)+1) / 2} \cdot q^{2 \nu-1}, \\
q^{\nu(3 \nu+1) / 2} & =q^{\nu(3 \nu-1) / 2} \cdot q^{\nu} .
\end{aligned}
$$

Besides the sparse and regular series expression, the $\eta$ function has a second crucial property that makes it well suited for computation: It is a modular form of weight $1 / 2$. As such, unlike $j$, it is not invariant under transformations in $\mathrm{Sl}_{2}(\mathbb{Z})$. 
However, its transformation behaviour is explicitly known (cf. [17, §4]) and easily computable. Thus, to obtain $\eta(z)$ for an arbitrary value of $z$, one should first transform $z$ into the fundamental domain $\mathcal{F}$, so that the series can be truncated at an exponent of order $O(n)$. Then the evaluation of $O(\sqrt{n})$ terms of the $\eta$ series for $O(h)$ distinct values (the constant being at most 4 for the $\mathfrak{w}_{p_{1}, p_{2}}$ mentioned in the beginning of this section) with a floating point precision of $O(n)$ digits can be carried out in time

$$
O(h \sqrt{n} M(n)),
$$

or

$$
O\left(|D|^{3 / 4} \log ^{2}|D| M\left(\sqrt{|D|} \log ^{2}|D|\right)\right) \subseteq O\left(|D|^{5 / 4} \log ^{5+\varepsilon}|D|\right)
$$

as $h \in O(\sqrt{|D|} \log |D|)$ and $n \in O\left(\sqrt{|D|} \log ^{2}|D|\right)$ according to Section 4.

It remains, however, to verify that transforming the arguments into the fundamental domain is dominated by the cost of the series evaluation. The arguments being roots of an $N$-system $\left[A_{i}, B_{i}, C_{i}\right]$ with $A_{i},\left|B_{i}\right| \in O\left(N^{2} \sqrt{|D|}\right)=O(\sqrt{|D|})$, they may be transformed into $\mathcal{F}$ by reducing the quadratic forms in time $O(h M(\log |D|))$ (see [12, Prop. 5.4.3]), which is negligible. The same holds for arguments such as $\frac{z+1}{2}$ or $\frac{z}{N}$, corresponding to quadratic forms whose discriminants have absolute values in $O(|D|)$.

6.3. Multipoint evaluation. The algorithms of Sections 6.1 and 6.2 compute the values of modular functions one at a time; but for the sake of class polynomial computation, we need the values in many points. For polynomials, Algorithm 3.2 provides a fast way of doing exactly this. And indeed, from a numerical point of view a class invariant can be seen as a polynomial via its truncated $q$-expansion. Either one considers the function directly as done for $j$ in Section 6.1 or one proceeds via $\eta$ as in Section 6.2. In both cases, a polynomial of degree $O(n)$ has to be evaluated in $O(h)$ points, which by Algorithm 3.2 can be done in time

$$
\begin{aligned}
& O\left(\left(n \log n+h \log ^{2} h\right) M(n)\right) \subseteq O\left(\sqrt{|D|} \log ^{3}|D| M\left(\sqrt{|D|} \log ^{2}|D|\right)\right) \\
& \subseteq O\left(|D| \log ^{6+\varepsilon}|D|\right) .
\end{aligned}
$$

6.4. Newton iterations on the arithmetic-geometric mean. A new approach for evaluating modular functions in single arguments is described in [18]. It is based on the arithmetic-geometric mean and Newton iterations on a function involving it. The basic algorithm underlying [18, Theorem 4] computes the modular function $k^{\prime}$, whose square $\lambda$ satisfies

$$
\frac{256\left(1-\lambda+\lambda^{2}\right)^{3}}{(\lambda(1-\lambda))^{2}}=j .
$$

For an argument with imaginary part bounded by a constant and the precision $n$ tending to infinity, it has a complexity of $O(\log n M(n))$.

During class polynomial computations, the precision and the imaginary part of the arguments are tightly coupled, so that this algorithm is not sufficient to derive the desired complexity result. The modification of [18, Theorem 5] obtains the same complexity of $O(\log n M(n))$ uniformly in the argument. If the imaginary part of the argument is of the order of the required precision, then the algorithm of Section 6.2 or even the naïve algorithm of Section 6.1 already yield the desired result with a constant number of arithmetic operations. Otherwise, the argument 
is repeatedly divided by 2 until its imaginary part is smaller than a constant, which can be compensated by iterations of the arithmetic-geometric mean. Then the previous Newton algorithm converges sufficiently fast.

For other modular functions $f$, one may have the evaluation of $k^{\prime}$ followed by Newton iterations on the modular polynomial relating $k^{\prime}$ and $f$. For fixed $f$, this phase does not increase the complexity. The approach does not work, however, for $\eta$, which is a modular form of weight $1 / 2$ instead of a modular function (of weight 0 ). The algorithm of [18, Section 7.2] computes first $\theta_{00}^{2}$, a certain modular form of weight 1 , as the inverse of the arithmetic-geometric mean of 1 and $k^{\prime}$, and then $\eta$ as the twelfth root of $\lambda(1-\lambda) \theta_{00}^{2} / 16$ by a suitably initialised Newton process. Again, one obtains a complexity of $O(\log n M(n))$ for an evaluation at precision $n$, uniformly in the argument.

The complexity for evaluating in $h$ arguments then becomes

$$
O(h \log n M(n)) \subseteq O\left(\sqrt{|D|} \log ^{2}|D| M\left(\sqrt{|D|} \log ^{2}|D|\right)\right) \subseteq O\left(|D| \log ^{5+\varepsilon}|D|\right)
$$

with the estimates of Section 4 for $h$ and $n$. Taking into account the time needed to compute the class polynomial from its roots by Algorithm 3.1, this proves Theorem 1.1.

\section{IMPLEMENTATiON}

The algorithms of this article have been implemented using gmp 32 with an assembly patch for 64 bit AMD processors 29, mpfr 33 and mpc 24, for the multiprecision arithmetic and mpfrcx [20] for the polynomial operations. Table 1$]$ provides running times for class numbers between 2500 and 100000 , obtained on an AMD Opteron 250 with $2.4 \mathrm{GHz}$. All timings are given in seconds and rounded to two significant digits. (The computations for class number 100000 have been carried out on a $2.2 \mathrm{GHz}$ machine disposing of more memory, and the running times have been scaled accordingly.) For each class number, the discriminant with smallest absolute value has been chosen. Only the algorithms of Sections 6.2 to 6.4 are taken into account; the naïve approach of Section 6.1 is clearly inferior to the one exploiting the sparsity of $\eta$. The chosen class invariant is the double $\eta$-quotient $\mathfrak{w}_{3,13}$, and its values are obtained by precomputing a table for the values of $\eta$ at the $h$ reduced quadratic forms.

The first lines of the table provide some general information. The precision (1) of the floating point computations is obtained by increasing the estimate of Section 4 by $1 \%$ to account for potential rounding errors. As the chosen class invariant is not $j$, a correction factor depending on the class invariant needs to be used; see Section 4. This factor is correct only asymptotically, which explains why the actual height (2) is a bit smaller than the precision estimate. For $j$, the two are closer to each other. $M(n)$ is measured by computing the first 100000 successive powers of $\pi+i \gamma$ with Euler's constant $\gamma$.

The second block of lines provides timings for the steps that are independent of the algorithm used for evaluating the modular function. As can be seen, the class group computation (4) is completely negligible. Line (5) corresponds to the effort of deriving all values of the class invariant from the tabulated $\eta$ values (reduction of quadratic forms, multiplication by 24-th roots of unity and computation of the $\eta$ quotients). The computation of the polynomial from its roots (6) corresponds 
TABLE 1. Running times

\begin{tabular}{ll|r|r|r|r|r|} 
& & 5000 & 10000 & 20000 & 40000 & 100000 \\
\hline & $|D|$ & 6961631 & 23512271 & 98016239 & 357116231 & 2093236031 \\
$(1)$ & precision $n$ (bits) & 9540 & 20317 & 45179 & 96701 & 264727 \\
$(2)$ & height (in base 2) & 8431 & 18114 & 40764 & 87842 & 242410 \\
$(3)$ & $M(n)$ & 7.3 & 23 & 75 & 230 & 1080 \\
\hline$(4)$ & class group & 0.1 & 0.1 & 0.4 & 1.3 & 6.8 \\
$(5)$ & conjugates from $\eta$ & 3.4 & 21 & 140 & 890 & 10000 \\
$(6)$ & poly. from roots & 13 & 93 & 730 & 5200 & 120000 \\
\hline & sparse series & & & & & \\
$(7)$ & $\eta$ & 12 & 98 & 900 & 7700 & 140000 \\
$(8)$ & of which $q_{i}$ & 3.0 & 22 & 170 & 1300 & 20000 \\
$(9)$ & total time & 28 & 210 & 1800 & 14000 & 270000 \\
\hline & multipoint eval. & & & & & \\
$(10)$ & $\eta$ & 93 & 640 & 5700 & 42000 & aborted \\
$(11)$ & total time & 110 & 750 & 6500 & 48000 & \\
\hline & $(10) /(7)$ & 7.8 & 6.5 & 6.3 & 5.5 & - \\
\hline & AGM & & & & & \\
$(12)$ & $\eta$ & 32 & 200 & 1400 & 9900 & 130000 \\
$(13)$ & total time & 48 & 320 & 2300 & 16000 & 260000 \\
\hline & $(12) /(7)$ & 2.7 & 2.0 & 1.6 & 1.3 & 0.93 \\
\hline
\end{tabular}

precisely to Algorithm 3.1 and provides a measure for the complexity of the operations with polynomials. For constructing the largest polynomial of degree 100000, the polynomial FFT has been disabled during the last steps and replaced by ToomCook multiplication, since the FFT consumed too much memory; this explains the jump from $h=40000$.

The third block contains the timings for evaluating $\eta$ in the reduced quadratic forms (7) using the sparse series representation as described in Section 6.2, and the total running time for computing the class polynomials using this technique (9). Line (8) details the time spent in (7) (and also in (10)) for computing the $q_{i}$; it essentially measures the complex exponential.

The fourth block represents the corresponding results for the multipoint evaluation approach of Section 6.3, and the last block corresponds to the asymptotically fastest evaluation of Section 6.4, for which an implementation by Dupont has been used. As explained in Section 6.4, the algorithm requires to switch to the sparse series evaluation when the imaginary part of the argument becomes too large. In the implementation, the AGM code is disabled for an imaginary part larger than 5 .

Comparing first the evaluation of $\eta$ as a sparse series or by multipoint evaluation, one notices that the asymptotically faster algorithm is about 5 to 8 times slower on the examples and that it appears to catch up with growing class numbers. However, this happens so slowly that one cannot expect it to beat the algorithm in $O\left(|D|^{1.25+\varepsilon}\right)$ in the foreseeable future for any tractable instance.

The approach using Newton iterations on the AGM is faster than multipoint evaluation, but still hardly beats the asymptotically slower algorithm in $O\left(|D|^{1.25+\varepsilon}\right)$ : The biggest computed example of class number 100000 lies just beyond the crossover point! 
One notices that the growth rates of the running times of all algorithms, instead of behaving like $|D|$ or $|D|^{1.25}$, come closer to $|D|^{1.4}$ or $|D|^{1.6}$. A small part of this can be explained by the rather peculiar choice of discriminants. Taking the first one with a given class number, the precision $n$ is rather large compared to $h$ and $\sqrt{|D|}$, since there are many forms with small $A$ so that the sum $\sum_{i=1}^{h} \frac{1}{A_{i}}$ becomes comparatively large.

However, the major reason for the faster than predicted growth of computing time is that for the floating point arithmetic the range for asymptotically fast algorithms is not yet reached. The threshold for switching to the FFT in gmp on the test machine is set to about 500000 bits; so the examples still lie in the Karatsuba or Toom-Cook range, which accounts for a growth of $M(n)$ of $n^{\log 3 / \log 2}$, respectively, $n^{\log 5 / \log 3}$ instead of $n$.

A possible improvement of the multipoint evaluation approach consists of conveniently grouping the arguments. For instance, in the example of class number 5000 , the function $\eta$ has to be evaluated in 2501 arguments (corresponding to the two ambiguous forms and 2499 pairs of opposite non-ambiguous forms). Assuming the worst case of $|q| \approx e^{-\pi \sqrt{3}}$, which is almost reached for the largest values of $A$, one can approximate $\eta$ by a polynomial of degree 1190. As multipoint evaluation should be most efficient when the number of arguments is about half the degree, it makes sense to perform four evaluations in 625, respectively, 626 arguments each. But sorting them by their absolute values, the smaller ones do not actually require such a high degree approximation of $\eta$ : In the example, an approximation of degree $260,551,805$, respectively, 1190 is sufficient for the four chunks of $q$. Then the time used for multipoint evaluation drops from 93s to 64s. Experimenting with different partitions of the arguments (three, respectively, five parts of the same size, parts of different sizes adapted to the degree of the approximations, etc.) yields similar results, far from competing with the sparse series evaluation.

Another point to take into account is the space requirements of the algorithms. When each root of the class polynomial is computed separately, only $O(h n)$ bits need to be stored, which is linear in the output size. Multipoint evaluation as described in Section 3.2, however, requires that the tree constructed in Step 1 of Algorithm 3.2 be maintained in memory, so that the occupied space grows by a logarithmic factor to reach $O(h n \log h)$. This logarithmic factor could be saved by evaluating in chunks of $O\left(\frac{h}{\log h}\right)$ arguments, as explained in Lemma 2.1 of [28].

Anyway, the example class polynomial of degree 100000 uses over 5 GB as an uncompressed text file and is computed in about 3 days. This shows that the limiting factor is the memory requirement rather than the running time, as can be expected from algorithms that have a close to linear complexity with respect to their output size.

As a final remark, one notices that the algorithms behave numerically well, even though rounding errors do occur during floating point computations. For the algorithm of Section 6.2. this can be explained by the sparsity of the $\eta$ series and the fact that all coefficients are +1 and -1 . Indeed, if the last few digits of a term are erroneous, these errors propagate to subsequent terms. However, the absolute magnitude of such errors decreases rapidly, so that the wrong digits in later terms actually do not intervene in the additions. (Otherwise said, the computations may as well be carried out with fixed point numbers, and indeed a simulation of fixed point arithmetic using floating point numbers of decreasing precision yields accurate results.) 


\section{COMPARISON TO OTHER APPROACHES}

8.1. Chinese remaindering. In [1], the authors suggest an approach for directly computing class polynomials for $j$ modulo a prime $p$. The basic idea is to derive the polynomial modulo many small primes by enumerating all elliptic curves modulo these small primes and only retaining those having complex multiplication by $\mathcal{O}_{D}$. Then a Chinese remainder technique allows us to obtain the class polynomial modulo $p$.

Unfortunately, it is not sufficient to gather only $O(\log p)$ bits of information modulo small primes per coefficient of the class polynomial, although this is the information contained in the final output. In fact, so many small primes are needed that the class polynomial could be reconstructed over $\mathbb{Z}$ instead of only modulo $p$. The complexity derived in Section 3.2 of [1] is

$$
O\left(|D|^{3 / 2} \log ^{10}|D|+|D| \log ^{2}|D| \log p+\sqrt{|D|} \log ^{2} p\right),
$$

and already the term depending only on $|D|$ is worse than what is obtained with the algorithms of Section 6 .

Thus, it is asymptotically faster to compute the class polynomial over $\mathbb{Z}$ using floating point approximations and to reduce it modulo $p$ afterwards.

8.2. $p$-adic algorithms. Couveignes and Henocq suggest in [13] a $p$-adic approach for computing class polynomials for $j$. The basic idea is to look for a small prime $p$ and (by an exhaustive search) an elliptic curve modulo $p$ with complex multiplication by $\mathcal{O}_{D}$. This curve is then lifted to the $p$-adic numbers with a high enough precision so that the class polynomial may be reconstructed. Alternatively, they show how to work with supersingular curves. The complexity of their approach is $O\left(|D|^{1+\varepsilon}\right)$, where the exact power of the logarithmic factor has not been worked out. By nature, the algorithm is not affected by rounding errors, and using the explicit bound of Theorem 1.2 its output is certified to be correct.

So the complex and the $p$-adic approach are both essentially linear in the size of the class polynomials. All variants have been implemented (for more details on the complex implementation, see [21; for the ordinary $p$-adic algorithm, see [10; for the supersingular one, 35]), and all seem to work reasonably well in practice. The floating point algorithms are easy to implement with arbitrary class invariants using the results of 38 . The $p$-adic approach with ordinary curves has been made to work with certain class invariants other than $j$; see [10] and [9, Chapter 6]. The

considerable overhead involved makes it unclear whether it is competitive with the complex approach.

\section{ACKNOWLEDGMENTS}

I am grateful to Régis Dupont for letting me use his code to test the fast evaluation of $\eta$ via the arithmetic-geometric mean. I thank Karim Belabas for pointing out to me the basic idea of Section 4. Pierrick Gaudry and François Morain for numerous discussions and Dan Bernstein, Reinier Bröker, Kristin Lauter and Marco Streng for helpful comments. 


\section{REFERENCES}

1. Amod Agashe, Kristin Lauter, and Ramarathnam Venkatesan, Constructing elliptic curves with a known number of points over a prime field, High Primes and Misdemeanours: Lectures in Honour of the 60th Birthday of Hugh Cowie Williams (Alf van der Poorten and Andreas Stein, eds.), Fields Institute Communications, vol. 41, American Mathematical Society, 2004. MR2075643 (2005m:11112)

2. A. O. L. Atkin and D. J. Bernstein, Prime sieves using binary quadratic forms, Mathematics of Computation 73 (2004), no. 246, 1023-1030. MR2031423 (2004i:11147)

3. A. O. L. Atkin and F. Morain, Elliptic curves and primality proving, Mathematics of Computation 61 (1993), no. 203, 29-68. MR1199989 (93m:11136)

4. Eric Bach, Explicit bounds for primality testing and related problems, Mathematics of Computation 55 (1990), no. 191, 355-380. MR1023756 (91m:11096)

5. Paulo S. L. M. Barreto, Ben Lynn, and Michael Scott, Constructing elliptic curves with prescribed embedding degrees, Security in Communication Networks - Third International Conference, SCN 2002, Amalfi, Italy, September 2002 (Berlin) (Stelvio Cimato, Clemente Galdi, and Giuseppe Persiano, eds.), Lecture Notes in Computer Science, vol. 2576, SpringerVerlag, 2003, pp. 257-267.

6. Richard P. Brent, Fast multiple-precision evaluation of elementary functions, Journal of the ACM 23 (1976), no. 2, 242-251. MR0395314 (52:16111)

7. Friederike Brezing and Annegret Weng, Elliptic curves suitable for pairing based cryptography, Designs, Codes and Cryptography 37 (2005), no. 1, 133-141. MR2165045 (2006e:14029)

8. N. Brisebarre and G. Philibert, Effective lower and upper bounds for the Fourier coefficients of powers of the modular invariant $j$, Journal of the Ramanujan Mathematical Society 20 (2005), 255-282. MR2193216 (2006k:11074)

9. Reinier Bröker, Constructing elliptic curves of prescribed order, Proefschrift, Universiteit Leiden, 2006.

10. Reinier Bröker and Peter Stevenhagen, Elliptic curves with a given number of points, Algorithmic Number Theory - ANTS-VI (Berlin) (Duncan Buell, ed.), Lecture Notes in Computer Science, vol. 3076, Springer-Verlag, 2004, pp. 117-131. MR2137348 (2005m:11113)

11. Michele Cipolla, Un metodo per la risoluzione della congruenza di secondo grado, Napoli Rend. 9 (1903), 153-163.

12. Henri Cohen, A course in computational algebraic number theory, Graduate Texts in Mathematics, Springer-Verlag, New York, 1993. MR1228206 (94i:11105)

13. Jean-Marc Couveignes and Thierry Henocq, Action of modular correspondences around CM points, Algorithmic Number Theory - ANTS-V (Berlin) (Claus Fieker and David R. Kohel, eds.), Lecture Notes in Computer Science, vol. 2369, Springer-Verlag, 2002, pp. 234-243. MR2041087 (2005b:11077)

14. David A. Cox, Primes of the form $x^{2}+n y^{2}-$ Fermat, class field theory, and complex multiplication, John Wiley \& Sons, New York, 1989. MR1028322 (90m:11016)

15. R. Dedekind, Erläuterungen zu den vorstehenden Fragmenten, Bernhard Riemann's gesammelte mathematische Werke und wissenschaftlicher Nachlaß (R. Dedekind and H. Weber, eds.), Teubner, Leipzig, 1876, pp. 438-447.

16. Max Deuring, Die Typen der Multiplikatorenringe elliptischer Funktionenkörper, Abhandlungen aus dem mathematischen Seminar der hamburgischen Universität 14 (1941), 197-272. MR0005125 (3:104f)

17. - Die Klassenkörper der komplexen Multiplikation, Enzyklop. d. math. Wissenschaften, vol. I 2 Heft 10, Teubner, Stuttgart, 2e ed., 1958. MR0167481 (29:4754)

18. Régis Dupont, Fast evaluation of modular functions using Newton iterations and the AGM, To appear in Mathematics of Computation, http://www.lix.polytechnique.fr/Labo/Regis . Dupont/preprints/Dupont_FastEvalMod.ps.gz 2007.

19. Régis Dupont, Andreas Enge, and François Morain, Building curves with arbitrary small MOV degree over finite prime fields, Journal of Cryptology 18 (2005), no. 2, 79-89. MR 2148052 (2006c:11073)

20. Andreas Enge, mpfrcx - a library for univariate polynomials over arbitrary precision real or complex numbers, Version 0.1, http://www.lix.polytechnique.fr/Labo/Andreas.Enge/ Software.html 
21. Andreas Enge and François Morain, Comparing invariants for class fields of imaginary quadratic fields, Algorithmic Number Theory - ANTS-V (Berlin) (Claus Fieker and David R. Kohel, eds.), Lecture Notes in Computer Science, vol. 2369, Springer-Verlag, 2002, pp. 252266. MR2041089 (2005a:11179)

22. — Further investigations of the generalised Weber functions, In preparation, 2007.

23. Andreas Enge and Reinhard Schertz, Constructing elliptic curves over finite fields using double eta-quotients, Journal de Théorie des Nombres de Bordeaux 16 (2004), 555-568. MR2144957 (2006d:11063)

24. Andreas Enge and Paul Zimmermann, mpc - a library for multiprecision complex arithmetic with exact rounding, Version 0.4.6, http://www.lix.polytechnique.fr/Labo/Andreas.Enge/ Software.html

25. Leonhard Euler, Evolutio producti infiniti $(1-x)(1-x x)\left(1-x^{3}\right)\left(1-x^{4}\right)\left(1-x^{5}\right)\left(1-x^{6}\right)$ etc. in seriem simplicem, Acta academiae scientiarum Petropolitanae 1780:I (1783), 125-169, Opera Omnia I.3:472-479.

26. Martin Fürer, Faster integer multiplication, Proceedings of the 39th Annual ACM Symposium on Theory of Computing - STOC'07 (New York) (Association for Computing Machinery, ed.), ACM, 2007, pp. 57-66.

27. Joachim von zur Gathen and Jürgen Gerhard, Modern computer algebra, Cambridge University Press, 1999. MR1689167 (2000j:68205)

28. Joachim von zur Gathen and Victor Shoup, Computing Frobenius maps and factoring polynomials, Computational Complexity 2 (1992), 187-224. MR.1220071 (94d:12011)

29. Pierrick Gaudry, Assembly support for GMP on AMD64, April 2005, http://www.loria.fr/ ¿gaudry/mpn_AMD64/

30. Alice Gee, Class invariants by Shimura's reciprocity law, Journal de Théorie des Nombres de Bordeaux 11 (1999), no. 1, 45-72. MR1730432 (2000i:11171)

31. Alice Gee and Peter Stevenhagen, Generating class fields using Shimura reciprocity, Algorithmic Number Theory - ANTS-III (Berlin) (J. P. Buhler, ed.), Lecture Notes in Computer Science, vol. 1423, Springer-Verlag, 1998, pp. 441-453. MR.1726092 (2000m:11112)

32. Torbjörn Granlund et al., gmp - GNU multiprecision library, Version 4.2.1, http://gmplib. org/

33. Guillaume Hanrot, Vincent Lefèvre, Patrick Pélissier, and Paul Zimmermann et al., mpfr - $a$ library for multiple-precision floating-point computations with exact rounding, Version 2.2.1, http://www.mpfr.org

34. A. K. Lenstra and H. W. Lenstra Jr., Algorithms in number theory, Algorithms and Complexity (Jan van Leeuwen, ed.), Handbook of Theoretical Computer Science, vol. A, Elsevier, Amsterdam, 1990, pp. 673-715. MR.1127178

35. R. Lercier and E. Riboulet-Deyris, Elliptic curves with complex multiplication, Posting to Number Theory List, http://listserv.nodak.edu/cgi-bin/wa.exe?A2=ind0401\& L=nmbrthry\&P=R305, 2004 .

36. Atsuko Miyaji, Masaki Nakabayashi, and Shunzou Takano, New explicit conditions of elliptic curve traces for FR-reduction, IEICE Trans. Fundamentals E84-A (2001), no. 5, 1234-1243.

37. Reinhard Schertz, Die singulären Werte der Weberschen Funktionen $\mathfrak{f}_{,} \mathfrak{f}_{1}, \mathfrak{f}_{2}, \gamma_{2}, \gamma_{3}$, Journal für die reine und angewandte Mathematik 286/287 (1976), 46-74. MR0422213 (54:10205)

38. Weber's class invariants revisited, Journal de Théorie des Nombres de Bordeaux 14 (2002), no. 1, 325-343. MR1926005 (2003j:11139)

39. A. Schönhage and V. Strassen, Schnelle Multiplikation großer Zahlen, Computing 7 (1971), 281-292. MR0292344 (45:1431)

40. Arnold Schönhage, Fast reduction and composition of binary quadratic forms, Proceedings of the 1991 International Symposium on Symbolic and Algebraic Computation - ISSAC '91 (Stephen M. Watt, ed.), ACM Press, 1991, pp. 128-133.

41. René Schoof, The exponents of the groups of points on the reductions of an elliptic curve, Arithmetic Algebraic Geometry (Boston) (G. van der Geer, F. Oort, and J. Steenbrink, eds.), Birkhaüser, 1991, pp. 325-335. MR.1085266 (91j:11043) 
42. Carl Ludwig Siegel, Über die Classenzahl quadratischer Zahlkörper, Acta Arithmetica 1 (1936), 83-86.

43. Heinrich Weber, Lehrbuch der Algebra, 2nd ed., vol. 3: Elliptische Funktionen und algebraische Zahlen, Vieweg, Braunschweig, 1908.

inRIa Saclay-Île-de-France \& Laboratoire D'Informatique (CNRS/UMR 7161), École polytechnique, 91128 Palaiseau Cedex, France

E-mail address: enge@lix.polytechnique.fr 